\title{
Condutas do enfermeiro(a) na prevenção do câncer de próstata: uma revisão bibliográfica
}

\author{
Nurses' conduct in the prevention of prostate cancer: a literature review \\ Conducta de las enfermeras en la prevención del cáncer de próstata: revisión de la \\ literatura
}

Marcela Gonçalves da Silva ${ }^{1 *}$, Simone Cristina da Silva Melo ${ }^{1}$, Francisca Moreira da Silva ${ }^{1}$, Maria Gorete Repolho Laurido ${ }^{1}$, Joyciane Rodrigues da Silva ${ }^{1}$, Deyse Lima de Azevedo ${ }^{1}$, Mauro Souza dos Santos ${ }^{1}$, Leandrio Taveira de Paula1', Denizângela Martins Pinto' ${ }^{1}$, Graciana de Sousa Lopes¹.

\section{RESUMO}

Objetivo: Verificar a importância da atuação do Enfermeiro dentro da política nacional do homem na prevenção do câncer da próstata. Revisão bibliográfica: Segundo um levantamento feito pelo Ministério da Saúde, cerca de um terço (31\%) dos homens brasileiros não se consulta com médicos regularmente para acompanhar seu estado de saúde pois acreditam em sua virilidade e que não ficam doentes por simplesmente serem fortes e a porcentagem de uma enfermidade os alcança-los é bem reduzida devido a esses motivos. A enfermagem é uma profissão que tem um papel fundamental no processo de educação em saúde, esclarecendo dúvidas, promovendo estratégias educativas de prevenção de agravos à saúde e incentivando o cuidado da população masculina. Considerações finais: A aplicação do projeto terapêutico tem como meta intervir em prol do alcance do objetivo principal que é mobilizar ações e serviços de saúde que estimulem a população masculina a cuidar mais de sua saúde.

Palavras-chave: Enfermagem, Câncer de próstata, Prevenção primária.

\begin{abstract}
Objective: To verify the importance of the role of the Nurse within the national policy of men in the prevention of prostate cancer. Bibliographic review: According to a survey carried out by the Ministry of Health, about a third $(31 \%)$ of Brazilian men do not regularly consult with doctors to monitor their health status as they believe in their virility and that they do not get sick simply because they are strong and the percentage of a disease reaching them is very low due to these reasons. Nursing is a profession that has a fundamental role in the health education process, clarifying doubts, promoting educational strategies for the prevention of health problems and encouraging the care of the male population. Final considerations: The application of the therapeutic project aims to intervene in order to achieve the main objective, which is to mobilize health actions and services that encourage the male population to take better care of their health.
\end{abstract}

Key words: Nursing, Prostate cancer, Primary prevention.

\section{RESUMEN}

Objetivo: Verificar la importancia del rol de la Enfermera dentro de la política nacional del hombre en la prevención del cáncer de próstata. Revisión bibliográfica: Según una encuesta realizada por el Ministerio de Salud, alrededor de un tercio (31\%) de los hombres brasileños no consultan regularmente a los médicos para controlar su estado de salud porque creen en su virilidad y que no se enferman simplemente porque son fuertes y el porcentaje de enfermedad que les llega es muy bajo por estas razones. La enfermería es una profesión que tiene un papel fundamental en el proceso de educación en salud, aclarando dudas, promoviendo estrategias educativas para la prevención de problemas de salud y fomentando el cuidado de la población masculina. Consideraciones finales: La aplicación del proyecto terapéutico pretende intervenir para lograr el objetivo principal, que es movilizar acciones y servicios de salud que incentiven a la población masculina a cuidar mejor su salud.

Palabras clave: Enfermería, Cáncer de próstata, Prevención primaria.

\footnotetext{
${ }^{1}$ Centro Universitário Fametro. Manaus - AM. *E-mail: marcelagoncalves.ms@gmail.com
} 


\section{INTRODUÇÃO}

O câncer é uma das principais causas de morte no mundo, estimando-se atingir mais de 21 milhões de pessoas até 2030. O número de pessoas que morrem com câncer é duas vezes maior que o número de pessoas que morrem por complicações relacionadas ao Vírus da Imunodeficiência Humana/ Síndrome da Imunodeficiência Adquirida (HIV/AIDS), tuberculose e malária combinadas. Com a evolução dessa patologia observa-se a necessidade de aperfeiçoar os cuidados da equipe de saúde que atuem nessas neoplasias. A má formação celular em que ocorre alteração permanente e hereditária nas células, resulta em proliferações patológicas excessivas de tecidos e órgãos, não intencional, que podem ser classificadas como benignas e malignas (BRASIL, 2017).

De acordo com o Minitério da Saúde (MS) define que o Câncer de Próstata é mais comum em homens da terceira idade, sendo este um dos principais causadores de óbitos. No Brasil este é o câncer de maior incidência em homens, perdendo apenas para o câncer de pele não-melanoma. Sendo assim de acordo com o Instituto Nacional do Câncer (INCA) (2018) foram 13.772 mortes da classe masculina em 2013 e a estimativa de casos novos de Câncer de Próstata para 2020 é 68.220 (BRASIL, 2017).

Segundo Vieira CG (2012) refere que o Sistema Único de Saúde (SUS), atualmente indica que a população masculina realize regularmente de forma preventiva os exames de: Dosage do Antígeno Prostático Específico (PSA), avaliação da próstata, através do exame de toque retal realizado por um especialista e a ultrassonografia transretal, sendo assim quando esta população ainda possui muito preconceito ao ouvi a palavra toque retal, deixando dessa forma de realizar este exame preventivo capaz de detectar precocemente o câncer.

Sendo assim, de acordo com Minitério da Saúde (MS) informa que o câncer age de forma silenciosa no início, sem apresentar sintomas aparentes, e assim evolui rapidamente para o estágio avançado da doença, podendo ter com sintomas nesta fase a dor lombar, dificuldade de urinar, aumento da frequência da micções e a presença de sangue ou sêmen na urina (BRASIL, 2018).

Dessa forma, na intenção de que o homem fosse em busca por exames e o cuidar da saúde, o Ministério da Saúde (MS) implantou no ano de 2008, a Política Nacional de Atenção Integrada à Saúde do Homem (PNAISH), este surgiu juntamente com a Política Nacional de Atenção Básica (PNAB), visto que os objetivos principais são: qualificar a assistência a saúde masculina na perspectiva de linha de cuidado de forma que se tenha resguardo a integralidade, além de qualificar a atenção primária, de forma que esta não se restrinja a atender apenas a recuperação e sim a promoção e a prevencao de agravos (FURTADO MS, et al., 2012; VIEIRA KLD, 2013).

Dessa maneira, por ser um assunto peculiar e de grau emergente, o homem com câncer de próstata requer do profissional da saúde, especialmente do enfermeiro, atenção especial para o aconselhamento de medidas preventivas e curativas, objetivando alcançar a promoção e a manutenção da sua saúde (BELINELO RGS, et al., 2014).

Diante destas considerações e da carência de produção científica sobre a temática abordada, justifica-se a realização do presente estudo, que tem como objetivo identificar as evidências científicas produzidas disponíveis na literatura sobre a assitência de enfermagem ao homem com câncer de próstata.

\section{REVISÃO BIBLIOGRÁFICA}

\section{Câncer de próstata}

Segundo um levantamento feito pelo Ministério da Saúde, cerca de um terço $(31 \%)$ dos homens brasileiros não se consulta com médicos regularmente para acompanhar seu estado de saúde pois acreditam em sua virilidade e que não ficam doentes por simplesmente serem fortes e a porcentagem de uma enfermidade os alcançá-los é bem reduzida devido a esses motivos. Bem, pelo menos é essa a concepção de muitos homens (BRASIL, 2010). O sucesso da Política Nacional de Atenção Integral à Saúde do Homem não se traduz somente por sua implantação, mas principalmente pela atuação de profissionais qualificados para 0 
atendimento da população masculina. No entanto, nota-se que as mudanças realizadas se refletem no aumento do número de profissionais homens atuantes no setor e na criação de serviços específicos para tal público (BARBOSA CJ, 2014).

Segundo Bravo MS e Matos MCM (2009) refere que ainda assim nos tempos atuais o homem não busca ajuda, pelo fato de não aceitarem na maioria das vezes tratamento oriundos de médicos do mesmo sexo, ou pelo fato de acharem que a masculinidade será colocada à prova em uma consulta com o especialista. $O$ primeiro paradoxo a ser pontuado refere-se à posição dos homens no processo de socialização que os coloca em lugar de destaque e privilégio na estrutura social. Nesse contexto, entende-se que os modelos de gênero (masculinidade) são incorporados como hábitos, estruturando as relações sociais. Embora essas categorizações sejam fruto de construções socioculturais, elas são percebidas pelos indivíduos como naturais, uma realidade ontológica.

Por todo o Brasil são promovidos eventos, palestras com foco na educação continuada e ações lembrando a importância sobre a prevenção e os cuidados que a população masculina precisa se conscientizar a respeito do diagnóstico precoce do câncer de próstata. Sendo assim, o mês de novembro definido como novembro Azul criado como uma forma para quebrar o preconceito que muitos homens têm em relação ao exame de toque (TORTORA GJ, DERRICKSON B, 2010).

Neste exame de toque, muitos não aceitam o fato de ter o ânus tocado e muito menos penetrado, mesmo que por um bom motivo, a campanha do novembro Azul vem justamente para quebrar esse preconceito e mostrar que compartilhar informações e levantar o tema são medidas necessárias. O Ministério da Saúde informa que durante o mês, há muito incentivo para a realização e identificação do câncer de próstata, seguido do exame de toque e o PSA ou antígeno prostático específico (exame de sangue) a fim de detectar o câncer na próstata (GOMES R, et al., 2008).

\section{Protocolo clínico: como se propaga o câncer da próstata}

A Próstata é uma glândula que tem uma estrutura física arredondada, medindo quatro centímetros de largura, dois centímetros de comprimento e três centímetros de cima para baixo, tem o tamanho aproximado de uma noz (TORTORA GJ, DERRICKSON B, 2010). Tem como função a produção de uma substância que junto com a secreção da vesícula seminal e dos espermatozoides formam o esperma (sêmen), sendo regulada por hormônios sexuais os "androgênios", sendo o mais importante deles a testosterona (FERREIRA UM, et al., 2007).

A função da próstata é produzir o fluido que protege e nutre os espermatozoides no sêmen, tornando-o mais líquido. Logo atrás da próstata, estão as glândulas denominadas vesículas seminais que produzem a maior parte do líquido para o sêmen. A uretra, que transporta a urina e o sêmen para fora do corpo através do pênis, atravessa o centro da próstata (ANKERST DP, et al., 2013).

O câncer não é uma doença, mas muitas, contudo, é significativo expor que quase todos os cânceres de próstata são adenocarcinomas, ou seja, se desenvolvem a partir das células glandulares. Outros tipos de câncer de próstata incluem sarcomas, carcinomas de pequenas células, tumores neuroendócrinos, carcinomas de células transicionais. Existem ainda outros tipos de câncer de próstata que são muito raros, onde podem crescer e se disseminar rapidamente, mas a maioria se desenvolve lentamente (DEVITA VT e LAWRENCE TS, 2015).

De fato, estudos de autópsia mostram que muitos homens mais velhos, e até mesmo alguns mais jovens e que morreram de outras doenças também tiveram câncer de próstata sem que nenhum sinal ou sintoma fosse apresentado, desconhecendo plenamente a existência da doença em seu organismo (RHODEN EL e AVERBECK MA, 2009).

No que se refere ao CA de próstata este câncer é o sexto em todo mundo, e o que mais acomete o público masculino, chegando a cerca de $10 \%$ do total de câncer diagnosticado. Os sintomas iniciais do câncer de próstata na fase inicial, não provoca sinais de que o mesmo existe, porém, em estágio avançado este é capaz de causar alguns sintomas: micção frequente, fluxo urinário fraco ou interrompido, vontade de urinar de forma frequente, principalmente no período noturno, sangue presente na urina ou no sêmen, disfunção erétil, dor ou 
incômodo no quadril, pernas, coxas, costas, ombros ou outros ossos se caso a doença tiver se disseminado ou fraqueza ou dormência nos pés (INCA, 2017).

Tais sintomas clínicos do paciente podem ser comparados com exames de ultrassonografia trans retal, ressonância magnética, tomografia, ecografia, urografia, endoscopia urinária, biópsia, entre outros. Quanto a prevenção, do CA de próstata é a ação tomada para reduzir chances de contrair a doença, portanto prevenilo significa evitar fatores de risco que acabam aumentando as chances de desenvolver a enfermidade (INCA, 2017).

Parte da carga de prevenção do CA de próstata encontra-se com o indivíduo, que deve adotar comportamentos que minimizem o risco e a ocorrência da doença. A mortalidade por câncer de próstata é relativamente baixa, o que reflete, em parte, seu bom prognóstico quando diagnosticado e tratado oportunamente, por isso o diagnóstico tardio gera consequências negativas, a sobrevida média mundial estimada em cinco anos é de $58 \%$ (INCA, 2017).

Uma forma também de se prevenir a doença é cuidando da alimentação e está comprovado que uma dieta rica em frutas, verduras, legumes, grãos e cereais integrais, e com menos gordura, principalmente as de origem animal, ajuda a diminuir o risco de câncer, como também de outras doenças crônicas nãotransmissíveis, manter o peso corporal adequado, praticar atividade física. Nesse sentido, outros hábitos saudáveis também são recomendados, como fazer, no mínimo, 30 minutos diários de atividade física, manter o peso adequado à altura, diminuir o consumo de álcool e não fumar, entre os fatores que mais ajudam a prevenir o câncer de próstata estão: ter uma alimentação saudável, manter o peso corporal adequado, praticar atividade física, não fumar e evitar o consumo de bebidas alcóolicas (GOMES R, et al., 2008).

Nos países desenvolvidos, essa sobrevida passa para $76 \%$ e nos países em desenvolvimento $45 \%$, no Brasil o Sistema Público de Saúde vem disponibilizando à população masculina o exame de prevenção do câncer de próstata, mas a procura por este tipo de exame ainda é insignificante, devido o homem não ter o hábito de buscar os serviços de saúde, nem mesmo quando há queixas (VIEIRA LJE, et al., 2008).

Quando localizado apenas na próstata, o câncer de próstata pode ser tratado com cirurgia oncológica, radioterapia e até mesmo observação vigilante, em alguns casos especiais. No caso de metástase, ou seja, se o câncer da próstata tiver se espalhado para outros órgãos, a radioterapia é utilizada junto com tratamento hormonal, além de tratamentos paliativos (INCA, 2017).

A escolha do melhor tratamento é feita individualmente, por médico especializado, caso a caso, após definir quais os riscos, benefícios e melhores resultados para cada paciente, conforme estágio da doença e condições clínicas do paciente. Todas as modalidades de tratamento são oferecidas, de forma integral e gratuita, por meio do Sistema Único de Saúde (SUS) (QUIJADA PD, et al., 2008).

\section{Práticas que otimizam a assistência e prevenção ao câncer de próstata}

De acordo com INCA (2017) a Política Nacional da Atenção Básica voltada para os parâmetros e a saúde integral do homem tem por objetivo auxiliar, orientar e aprimorar os cuidados perante os índices de cura e mortalidade, permitindo assim o acompanhamento através do Política Nacional da Atenção Básica em busca da prevenção e qualidade de vida.

Desse modo, com a criação da PNAISH pelo Ministério da Saúde em 2009, cujo objetivo é facilitar e maximizar o acesso dos homens a este serviço estão descritas várias maneiras de aperfeiçoar a assistência oferecida pelo enfermeiro, para ocasionar uma mudança cultural, além de desenvolver esclarecimentos de dúvidas e incentivos a população masculina a realizar cuidados próprios (ALBANO BR, et al, 2010).

Para Furini AA, et al. (2017) torna-se fundamental não só falar sobre a temática ao paciente, mas também, propor formas de ampliar as informações a respeito do câncer de próstata e, principalmente mostrar a importância da prevenção e da necessidade de seguir as medidas preventivas acerca desta patologia. Segundo Depallens MA (2020) reforça que ao referir-se as altas prevalências do câncer de próstata, percebese que o de próstata é que mais acomete a população masculina. Ainda conforme os autores, a importância 
da orientação permite que o homem seja esclarecido quanto a promoção e prevenção dos riscos que corre ao não se cuidar, dessa forma obtém-se melhor qualidade de vida.

O câncer de próstata é agressivo a partir do momento em que as células cancerígenas vão ganhando espaço e a capacidade de invadir os tecidos vizinhos, desta forma tornando-se células assintomáticas. cuidados adequados devem ser iniciados e mantidos desde apresentação dos resultados até os cuidados a serem realizados em casa durante todo e qualquer diagnóstico (PINHEIRO JT, et al, 2015).

\section{A importância do enfermeiro no diagnóstico precoce de câncer de próstata}

O papel do enfermeiro perante aos cuidados assumem uma importância central perante as respostas às necessidades de cuidados destes pacientes, atuando na promoção saúde e detecção precoce de agravos, no sentido de orientá-los sobre os fatores de risco e medidas de prevenção (OLIVEIRA AJ, et al, 2015).

A importância da atuação do enfermeiro no tratar e cuidar da saúde dos homens, estes devem atentar para os aspectos culturais que os tornam singulares em âmbito geral, permitindo assim a continuidade do cuidado. Nesse sentido, é fundamental a discussão sobre a masculinidade para os serviços e profissionais de saúde, bem como para a população, no sentido de romper com o paradigma de invulnerabilidade dos homens e de fazer ecoar as necessidades desse grupo, muitas vezes, esquecidas e embutidas não só pelo sistema de saúde, mas pelo próprio homem (STORINO LP, et al., 2013).

As bases para intervenção em enfermagem devem-se manter sobre os pilares da educação em saúde, visto que devem desvelar o medo que os homens têm da prevenção, sendo assim necessário burlar estratégias que garantam de prevenção e detecção do câncer de próstata. O profissional enfermeiro como integrante da equipe multiprofissional e atuante na Estratégia Saúde Família (ESF), considerada porta de entrada para o sistema de saúde, apresenta papel de destaque, atuando mediante adoção de práticas assistenciais, preventivas e de promoção à saúde. Enfermagem tem papel fundamental na utilização do Processo de Enfermagem para viabilizar um atendimento organizado e de qualidade aos pacientes (CHAVES $R G$, et al., 2016).

\section{O papel da enfermagem no tratamento do câncer de próstata}

A enfermagem é uma profissão que tem um papel fundamental no processo de educação em saúde, esclarecendo dúvidas, promovendo estratégias educativas de prevenção de agravos à saúde e incentivando o cuidado da população masculina (ALBANO BR, et al., 2010). Esse profissional deve prestar assistência ao paciente, promovendo e mantendo a saúde do mesmo, criar vínculos com o paciente, proporcionando que o mesmo tenha um vínculo com eles de respeito, empatia e humanização, melhorando assim os cuidados biológicos e psicológicos do paciente (FRACOLLI LA, et al., 2012).

De acordo com Oguisso T, et al. (2018) o papel do enfermeiro junto ao paciente com câncer é muito importante, pois ele desempenha um papel na rotina e no cuidado contínuo desses pacientes que precisam tomar decisões e avaliar as intervenções que vêm sendo aplicadas e utilizar o cuidado sistematizado de forma organizada. Além do desenvolvimento de equipes interdisciplinares, métodos alternativos de tratamento também podem auxiliá-lo na última etapa (implantação do atendimento e avaliação do desfecho do óbito) para que os profissionais possam ampliar seus conhecimentos sobre a doença.

No que tange, para prestar segurança nos cuidados prestados aos pacientes, o profissional deve buscar evidências de intervenções já realizadas, avaliando de forma criteriosa os resultados obtidos pelos cuidados prestados em pacientes oncológicos prostáticos (OLIVEIRA AJR, et al., 2015). É neste contexto que entra a presença do enfermeiro, profissional tão importante no pré e pós tratamento posto que ele tem um amplo campo de trabalho para contribuir com a saúde do indivíduo em diversos tipos de serviços farmacêuticos, no entanto, a qualidade precisa ser administrada, e não há outra forma de prestar este serviço a não ser pela conscientização das pessoas, pois, por meio delas, identificaremos a causa das ocorrências e as ações corretivas para as mesmas e, para esse sucesso, é fundamental que haja comprometimento de todos envolvidos, desde a alta direção até os auxiliares, gerando assim uma liderança eficaz, um bom trabalho em equipe, qualidade e eficiência no serviço prestado (SILVA MOE, 2009). 
Dessa forma, na área da oncologia, o profissional qualificado é essencial no acompanhamento do paciente com câncer de próstata é extrema pois a integração de uma equipe com funcionamento harmônico, permite um ambiente mais seguro com amplos e efetivos canais de comunicação, torna os tratamentos mais efetivos, mais seguros e mais bem tolerados pelos pacientes, visto que há os que defendem a oncologia, por ser considerada uma atividade multiprofissional (RAMONE D, 2020).

Desvela-se que a equipe de enfermagem deve agir para evitar o pessimismo angustiante que geralmente se instala no convívio familiar com a vítima. Nos cuidados paliativos na oncologia, "o objetivo da assistência compreende na promoção da qualidade de vida e do conforto dos pacientes e da família que enfrentam juntos a enfermidade, atuando na prevenção e alívio dos sintomas e apoiando as necessidades psicossociais, emocionais e espirituais do enfermo e acompanhante (SILVA MMD, MOREIRA MC, 2011).

Ressalta-se que o enfermeiro tem que ter consciência da sua importância como o profissional do medicamento e do paciente assumindo o seu papel na sociedade, prestando atenção adequada, informando e orientando sobre o uso correto de cada medicamento, promovendo a saúde e melhorando a qualidade de vida, fazendo a diferença através da Atenção Farmacêutica, sendo assim, é muito importante que todo entendimento das informações constantes na prescrição (SILVA MOE, 2009).

\section{CONSIDERAÇÕES FINAIS}

A aplicação do projeto terapêutico tem como meta intervir em prol do alcance do objetivo principal que é mobilizar ações e serviços de saúde que estimulem a população masculina a cuidar mais de sua saúde. Tal objetivo, já explica por si só a importância do projeto. Quanto à relevância do projeto na intervenção, este possui papel esclarecedor a respeito do trabalho do Enfermeiro, capaz de promover conhecimento e sustentação a diversas teorias sobre a especificidade desta temática e do fazer profissional. Por sua vez os homens cuidam pouco da saúde pois os serviços públicos também não colaboram para este fim pois confiam sempre na perspectiva de gênero, a saúde do homem deve ser destacada não só para se desenhar o perfil epidemiológico da morbimortalidade masculina, portanto, os serviços de atenção básica costumam ser vistos como lugar de crianças, mulheres e idosos; e as ações de atenção básica voltadas para os segmentos masculinos ainda são tímidas.

\section{REFERÊNCIAS}

1. ALBANO BR, et al. Desafios para a inclusão dos homens nos serviços de atenção primária à saúde. Revista Enfermagem Integrada, 2010;3(2): 554-563.

2. ANKERST DP, et al. The impact of prostate volume, number of biopsy cores and American Urological Association symptom score on the sensitivity of cancer detection using the Prostate Cancer Prevention Trial risk calculator. The Journal of urology, 2013; 190(1): 70-76.

3. BARBOSA CJL. Saúde do homem na atenção primária: mudanças necessárias no modelo de atenção. Revista saúde e Desenvolvimento, 2014;6(3): 99-114.

4. BELINELO RGS, et al. Exames de rastreamento para o câncer de próstata: vivência de homens. Escola Anna Nery, 2014; 18(4): 697-704.

5. BRASIL. Ministério da Saúde. ABC DO SUS, Doutrinas e Princípios. DF: MS, 2010.

6. BRASIL. Ministério da Saúde. Instituto Nacional de Câncer José Alencar Gomes da Silva. Estimativa 2018: incidência de câncer no Brasil. Rio de Janeiro, INCA. 2017.

7. BRASIL. Ministério da Saúde. Secretaria Nacional de Assistência à Saúde. Instituto Nacional de Câncer. Câncer de próstata. Rio de Janeiro. INCA, 2018.

8. BRAVO MI, MATOS MCM. Social e sua relação com a Reforma Sanitária: elementos para o debate. Serviço Social e Saúde: Formação e trabalho profissional. 4. ed. São Paulo: Cortez. Brasília, DF.2009

9. CHAVES RG, et al. Perfil socioeconômico de homens em um Munícipio do Tocantins e sua percepção sobre toque retal e câncer de Próstata. Revista Saúde e Desenvolvimento, 2016;9(5), 37-56.

10. DEPALLENS MA, et al. Prevenção quaternária, reforma curricular e educação médica. Interface-Comunicação, Saúde, Educação, 2020; 24, 1-16.

11. DEVITA VT, LAWRENCE, TS. DeVita, Hellman, and Rosenberg's cancer: principles \& practice of oncology. Wolters Kluwer, 2008; 1897-1898.

12. FERREIRA UM, et al. Esteróides anabólicos androgênicos. Revista Brasileira em Promoção da Saúde, 2007;20(4): 267-275. 
13. FRACOLLI LA. et al. Instrumentos de avaliação da Atenção Primária à Saúde: revisão de literatura e metassíntese. Ciência \& Saúde Coletiva, 2014; 12(19): 4851-4860.

14. FURINI AA. Nível sérico de antígeno prostático específico em usuários de um laboratório clínico de Novo Horizonte. Revista Brasileira de Análises Clínicas, 2017; 49(1), 52-6.

15. FURTADO MS, et al. A saúde do homem na visão dos enfermeiros de uma unidade básica de saúde. Escola Anna Nery, 2012; 16(3): 561-568.

16. GOMES R, et al. A prevenção do câncer de próstata: uma revisão da literatura. Ciência \& Saúde Coletiva, 2008; 13(1): 235-246.

17. INSTITUTO NACIONAL DE CÂNCER JOSÉ ALENCAR GOMES DA SILVA (INCA). Estimativa 2017: detecção precoce: monitoramento das ações de controle do câncer de próstata. RIO DE JANEIRO: INCA; 2017.

18. OGUISSO T, et al. O exercício da enfermagem: uma abordagem ético-legal. In O exercício da enfermagem: uma abordagem ético-legal. 5. ed. Guanabara Koogan, 2018.

19. OLIVEIRA AJ. et al. A atuação da enfermagem frente às barreiras encontradas no diagnóstico precoce do câncer de próstata. Revista Eletrônica de Ciências Humanas, Saúde e Tecnologia, 2015;4(1), 29-65.

20. PINHEIRO JT. et al. Perfil dos homens participantes do ensaio comunitário sobre prevenção do câncer de próstata. Revista Bionorte, 2015; 4(1), 35-49.

21. QUIJADA PD, et al. Qualidade de vida relacionada à saúde de pacientes com câncer de próstata. Revista Cuidarte, 2017;8(3): 1826-1838.

22. RAMONE D, et al. Oncologia Clínica para o dia a dia. 1. ed. Rúbio Ltda, 2020.

23. RHODEN EL, AVERBECK MA. Câncer de próstata e testosterona: riscos e controvérsias. Arquivos Brasileiros de Endocrinologia \& Metabologia, 2009;53(8): 956-962.

24. SILVA MMD, MOREIRA MC. Sistematização da assistência de enfermagem em cuidados paliativos na oncologia: visão dos enfermeiros. Acta Paulista de Enfermagem, 2011; 24(2): 172-178.

25. SILVA MOE. Da exclusão à inclusão: concepções e práticas. Revista Lusófona de Educação, 2009;13:135-153.

26. STORINO LP, et al. Necessidades de saúde de homens na atenção básica: acolhimento e vínculo como potencializadores da integralidade. Escola Anna Nery, 2013; 17(4), 638-645.

27. TORTORA GJ, DERRICKSON B. Principios de anatomía y fisiología. Médica Panamericana, 2013.

28. VIEIRA CG, et al. O homem e o câncer de próstata: prováveis reações diante de um possível diagnóstico. Revista científica do ITPAC, 2012; 5(1): 1-9.

29. VIEIRA KLD, et al. Atendimento da população masculina em unidade básica saúde da família: motivos para a (não) procura. Escola Anna Nery Revista de Enfermagem, 2013; 17(1): 120-127.

30. VIEIRA LJE, et al. Prevenção do câncer de próstata na ótica do usuário portador de hipertensão e diabetes. Revista Ciência \& Saúde Coletiva, 2008; 13(1): 145-152. 